\section{(6) OPEN ACCESS}

- Additional material is published online only. To view please visit the journal online (http://dx.doi.org/10.1136/ gutjnl-2014-308252)

For numbered affiliations see end of article.

\section{Correspondence to Dr Patrick Tan, Duke-NUS Graduate Medical School, 11 Hospital Drive, Singapore 169610, Singapore; gmstanp@duke-nus.edu.sg Dr Heike I Grabsch, Maastricht University Medical Center Department of Pathology, P. Debyelaan 25, 6202 AZ Maastricht, The Netherlands; Heike.Grabsch@mumc.n}

Received 13 August 2014 Revised 8 September 2014 Accepted 9 September 2014 Published Online First 10 November 2014

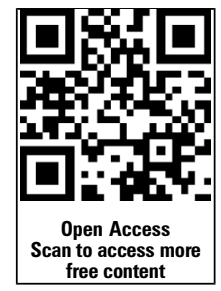

CrossMark

To cite: Lin SJ, GagnonBartsch $\mathrm{JA}$, Tan IB, et al. Gut 2015;64:1721-1731.

\title{
Signatures of tumour immunity distinguish Asian and non-Asian gastric adenocarcinomas
}

Suling J Lin, ${ }^{1}$ Johann A Gagnon-Bartsch, ${ }^{2}$ Iain Beehuat Tan, ${ }^{3}$ Sophie Earle, ${ }^{4}$ Louise Ruff, ${ }^{4}$ Katherine Pettinger, ${ }^{4}$ Bauke Ylstra, $^{5}$ Nicole van Grieken, ${ }^{5}$ Sun Young Rha, ${ }^{6}$ Hyun Cheol Chung, ${ }^{6}$ Ju-Seog Lee, ${ }^{7}$ Jae Ho Cheong, ${ }^{8}$ Sung Hoon Noh, ${ }^{8}$ Toru Aoyama, ${ }^{9}$ Yohei Miyagi, ${ }^{10}$ Akira Tsuburaya, ${ }^{1}$ Takaki Yoshikawa, ${ }^{9}$ Jaffer A Ajani, ${ }^{12}$ Alex Boussioutas, ${ }^{13,14}$ Khay Guan Yeoh, ${ }^{15,16}$ Wei Peng Yong, ${ }^{17}$ Jimmy So, ${ }^{18}$ Jeeyun Lee, ${ }^{19}$ Won Ki Kang, ${ }^{19}$ Sung Kim, ${ }^{20}$ Yoichi Kameda, ${ }^{21}$ Tomio Arai, $^{22}$ Axel zur Hausen, ${ }^{23,24}$ Terence P Speed, ${ }^{2,25,26}$ Heike I Grabsch, 4,23,24 Patrick Tan 1,27,28,29

\section{ABSTRACT}

Objective Differences in gastric cancer (GC) clinical outcomes between patients in Asian and non-Asian countries has been historically attributed to variability in clinical management. However, recent international Phase III trials suggest that even with standardised treatments, GC outcomes differ by geography. Here, we investigated gene expression differences between Asian and non-Asian GCs, and if these molecular differences might influence clinical outcome.

Design We compared gene expression profiles of 1016 GCs from six Asian and three non-Asian GC cohorts, using a two-stage meta-analysis design and a novel biostatistical method (RUV-4) to adjust for technical variation between cohorts. We further validated our findings by computerised immunohistochemical analysis on two independent tissue microarray (TMA) cohorts from Asian and non-Asian localities $(n=665)$.

Results Gene signatures differentially expressed between Asians and non-Asian GCs were related to immune function and inflammation. Non-Asian GCs were significantly enriched in signatures related to T-cell biology, including CTLA-4 signalling. Similarly, in the TMA cohorts, non-Asian GCs showed significantly higher expression of T-cell markers (CD3, CD45R0, CD8) and lower expression of the immunosuppressive T-regulatory cell marker FOXP3 compared to Asian GCs $(p<0.05)$. Inflammatory cell markers CD66b and CD68 also exhibited significant cohort differences $(p<0.05)$. Exploratory analyses revealed a significant relationship between tumour immunity factors, geographic localityspecific prognosis, and postchemotherapy outcomes. Conclusions Analyses of $>1600 \mathrm{GCs}$ suggest that Asian and non-Asian GCs exhibit distinct tumour immunity signatures related to T-cell function. These differences may influence geographical differences in clinical outcome, and the design of future trials particularly in immuno-oncology.

\section{INTRODUCTION}

Gastric cancer (GC) is a leading cause of global cancer mortality, with high incidence rates in Asia and parts of Latin America. ${ }^{1}$ Survival outcomes differ

\section{Significance of this study}

What is already known on this subject?

- Gastric cancer patients from different geographic localities are known to show different clinical outcomes.

- Conventionally, these clinical outcome differences have been attributed to differences in clinical management and disease stage.

- However, recent Phase III clinical trials with standardised treatment arms still demonstrated differences in gastric cancer outcomes by geographic locality.

\section{What are the new findings?}

- Our investigation of $>1600$ gastric cancers revealed that tumour immunity signatures differ significantly between Asian and non-Asian gastric cancers.

- Non-Asian gastric cancers were associated with enrichment of tumour infiltrating T-cells as well as T-cell gene expression signatures, including CTLA-4 signalling.

- Exploratory analysis suggests that these tumour immunity differences may contribute to geographical differences in clinical outcome.

How might it impact on clinical practice in the foreseeable future?

- The design of future gastric cancer trials, particularly in immuno-oncology, should consider tumour immunity differences in patients from different geographic localities, as they may impact on treatment response and clinical outcomes.

across geographical regions, with overall 5 -year survival rates being 10\%-15\% in North America ${ }^{2}$ and 45\%-50\% in East Asia. ${ }^{3}{ }^{4}$ These differences cannot be simply explained by improved early diagnosis in Asian countries, as they persist even after stratifying for disease stage. ${ }^{5}$ It has been suggested that these 
differences may reflect geographic variability in clinical practice. However, Asian patients treated in Western countries still exhibit superior outcomes compared with Caucasians, albeit worse than patients from Asian registries in home countries. ${ }^{6}$ Recently, a potentially deeper relationship between geographic locality and GC clinical outcome has emerged from international Phase III randomised trials, where the study populations and treatments are standardised across multiple countries. In the Avastin in Gastric Cancer (AVAGAST) trial $^{7}$ evaluating bevacizumab in advanced GC, subgroup analysis revealed a survival benefit in non-Asians but not in Asians. Conversely, in the Lapatinib Optimization Study in ErbB2 (HER2) Positive Gastric Cancer (LOGiC) trial ${ }^{8}$ benefit from lapatinib was observed in Asians but not non-Asians. Importantly, both studies were ultimately deemed 'negative' in the overall study population. It is thus important to investigate whether Asian and non-Asian GCs represent distinct molecular entities. This information may prove vital in selecting appropriate study populations for future trials.

Asians and non-Asian populations may differ in their GC risk factors. For example, Asian Helicobacter pylori strains are enriched in cagPAI pathogenicity gene variants compared with non-Asian strains, which may modulate carcinogenicity. ${ }^{9}$ Germline polymorphisms (eg, IL-1B, IL-1RN ${ }^{10}$ ) associated with GC risk also appear to differ across different populations. Regional variability in histopathological subtypes and tumour site locations have been reported, with intestinal subtype GC being particularly common in endemic countries while the incidence of diffuse subtype GC is less variable. ${ }^{11}$ However, somatic gene mutation and gene amplification rates in major cancer oncogenes such as KRAS, HER2 and FGFR2 appear to be similar in Asian and non-Asian GCs, ${ }^{12-15}$ suggesting that oncogenic pathways are broadly conserved between different populations. In summary, conflicting and overall very little data exist regarding whether Asian and non-Asian GCs are molecularly distinct.

Gene expression profiling technologies can provide 'molecular snapshots' of global transcriptional programmes expressed in tumours, not only from cancer cells but also from other tumourassociated cell types, such as fibroblasts, blood vessels and immune cells. To date, only one report (involving only 11 samples) has directly compared gene expression profiles between Eastern and Western GCs. ${ }^{16}$ Thus, a large study is warranted to clarify whether Asian and non-Asian GCs are molecularly distinct. In this hypothesis-generating study, we performed a two-stage meta-analysis integrating gene expression profiles of 1016 primary GCs from nine studies comprising Asian and non-Asian localities. We discovered and validated immune signature differences at the gene expression level between Asian and non-Asian GCs. The discovered gene expression differences were corroborated by subsequent identification of quantitative differences in intratumoural immune cells in two independent Asian and non-Asian GC cohorts. Exploratory survival analyses revealed that these tumour immunity factors significantly influenced geographic locality-specific survival.

\section{MATERIALS AND METHODS \\ GC patient cohorts}

Nine GC expression cohorts were analysed, involving 1016 patients (890 Asians and 126 non-Asians; online supplementary table S1). Studies were approved by Local Research Ethics Committees.

\section{Data preprocessing}

Platform-specific preprocessing steps were applied for Affymetrix and cDNA microarrays (detailed in online supplementary materials and methods). Two datasets (YGC and SamsungMC) were received in normalised form from collaborators. For these latter datasets, only summarisation of multiple probes mapping to the same gene was performed. All preprocessing steps were conducted within the statistical computing language R environment version 2.15 (http://www.r-project.org).

\section{Gene expression microarray analysis}

Due to technical variation across different microarray cohorts, gene expression measurements are often influenced not only by biological factors of interest (eg, geographic locality), but also by technical factors-including known/observed unwanted factors (eg, batch of hybridisation), and unknown/unobserved unwanted factors (eg, RNA degradation). We used RUV-4 ${ }^{17}$ (a new method similar to RUV-2) ${ }^{18}$ to reduce the impact of these unwanted factors (see online supplementary materials and methods). Briefly, RUV-4 uses 'negative control genes' (genes assumed to be uninfluenced by the biological factor of interest) to help identify the unwanted factors, which are then incorporated into a standard linear regression. In this study, housekeeping genes were used as controls (see online supplementary table $\mathrm{S} 2$ ). However, RUV-4 is distinct from standard normalisation methods using housekeeping genes, where gene expression values are adjusted using weights derived from differences in housekeeping gene levels. ${ }^{17}$ Specifically, to identify differentially expressed genes, $R U V-4$ fits a linear model on the expression data, accounting for known and unknown technical factors identified. In the process, two-group comparisons using empirical Bayes moderated t-statistics ${ }^{19}$ are made (eg, between 'Asian' and 'non-Asian' cohorts). Multiple hypothesis testing corrections were achieved using the Benjamini and Hochberg algorithm. Genes with adjusted $\mathrm{p}<0.05$ were considered to be differentially expressed. Ingenuity Pathway Analysis (IPA) and Gene-Set Enrichment Analysis (GSEA) was performed (online supplementary materials and methods) to identify expression modules and pathways distinct to Asian and non-Asian GCs. All top-ranked genesets were manually curated to confirm their accurate functional and pathway categorisations, and are presented in online supplementary table S3. For this study, GSEA was used largely for exploratory analyses, and instead of applying a stringent false discovery rate cut-off, we chose to analyse the top 10 genesets (ranked by normalised enrichment score) that were (1) associated with each of the localities and also (2) associated with low nominal $\mathrm{p}$ values (see online supplementary materials and methods).

\section{Immunohistochemistry and other tissue analyses}

Tissue microarrays (TMA) were constructed from an Asian GC cohort (Japanese; $n=219$ ) and a non-Asian cohort (Caucasian; $\mathrm{n}=446$ ). Slides from resection specimens were reviewed by four senior gastrointestinal pathology histopathologists (HG, TA, YM and $\mathrm{YK}$ ). A block with the highest tumour cell density was selected and marked for TMA construction. TMA sections were stained for immune cell markers CD66b (neutrophils), CD68 (pan-macrophages), CD45 (pan-leucocytes), CD3 (T-cells), CD8 (cytotoxic T-cells), CD45R0 (memory/activated T-cells) and FOXP3 (T-regulatory cells). TMA slides were subjected to automated image analysis as described previously. ${ }^{20}$ Wilcoxon Mann-Whitney tests were used to test whether particular markers were different between Japanese and Caucasian GCs, using tumour content-adjusted median immunohistochemical (IHC) values (significance $\mathrm{p}<0.05$ ). Epstein-Barr virus (EBV) RNA in situ hybridisation ${ }^{21}$ and assessment of the mismatch 
repair $(\mathrm{MMR})$ status $^{14}$ were also performed (see online supplementary methods).

\section{Statistical analyses}

Clinicopathological factors between Asian and non-Asian cohorts were compared using a two-tailed Welch's t-test. Survival analyses between Asian and non-Asian GCs were performed using the Kaplan-Meier method, with 5-year overall survival as the outcome metric, and log-rank tests to assess significance. Univariate and multivariate analyses were performed using Cox regression and Wald tests used to assess significance. Year of surgery was added to the multivariate model in order to account for potential influence of tissue block age on antigen recovery. For analyses involving CD68/CD3 ratios, samples were categorised into 'high' (3rd/4th quantiles) and 'low' (1st/2nd quantiles) groups, and a generalised linear model, using a gamma distribution, was fitted with CD68/CD3 ratios and significant covariates as the predictor variables and 5-year overall survival time as the response variable. One-tailed Welch's t-test was employed to test associations between CD68/CD3 ratios and geographic locality (significance threshold $\mathrm{p}<0.05$ ). CD68 and CD3 profiles from expression microarray datasets were also grouped into quantiles and CD68/CD3 ratios computed for all samples. Samples with a CD68/CD3 ratio greater than two were classified as 'high C68/CD3 ratio' and the remaining as 'low CD68/CD3 ratio'. Datasets with approximately $10 \%$ or a greater number of 'high CD68/CD3 ratio' samples (6 datasets) were included in further survival analyses. Survival curves and hazards were found to cross in all datasets analysed, hence supremum version of log-rank test (Renyi-type test) ${ }^{22}$ was used to assess significance of difference between curves. Due to nonproportional hazards, restricted mean survival times were computed at 1-year, 5-year and 10-year intervals.

\section{RESULTS}

Baseline characteristics of Asian and non-Asian GC datasets

We assembled nine independent GC microarray cohorts comprising 1016 tumour gene expression profiles-six from Asian localities (ie, HK_cDNA, Korea_SY, SamsungMC, SGset1, SGset2, YGC; $n=8 \overline{9} 0$ ), and three from non-Asian localities (ie, AMS_cDNA, AU_affy, LEEDS; n=126) (see online supplementary table S1). Most clinicopathologic parameters (eg, age, gender, stage etc.) were not significantly different between the Asian and non-Asian cohorts ( $p>0.05$; table 1$)$, with the exception of tumour location, where non-Asian cohorts had significantly more cases with tumours in the upper third of the stomach compared with Asian cohorts $(p=0.04)$. However, in agreement with the published literature, 5-year survival outcomes were significantly better in the Asian cohorts (figure 1).

\section{Two-stage meta-analysis}

Prior to combining the cohorts (gene expression data) for comparative analyses, it was necessary to reduce study-specific technical variation confounded with geographic locality (see online supplementary figure S3A). This could not be achieved via conventional methods (eg, dummy regression analysis). Hence, we used a novel algorithm, $R U V-4$, to reduce study-specific effects in the gene expression data, while retaining geographic localityspecific differences (see online supplementary figure S3B). However, despite RUV-4 being able to reduce study-specific effects in the gene expression data, it cannot eliminate them entirely, and errors at the individual gene level can still persist even after correction. The potential presence of residual confounding prompted us to compare the Asian and non-Asian
GCs at the gene signatures level (multiple genes) rather than single genes. We also employed a two-stage meta-analysis design to further reduce platform-specific differences (figure 2). In Stage 1, the analysis was restricted to 'Affymetrix' datasets $(n=299)$ since examination of the mean profile pairs-plot for each dataset suggests that 'Affymetrix' datasets were most comparable (see online supplementary figure S4). In Stage 2, the assessment of differential gene signatures between Asian and non-Asian GCs identified in Stage 1 also showed similar differential enrichment in the noisier 'non-Affymetrix' datasets $(n=717)$.

\section{Distinct expression signatures in Asian and non-Asian GCs (Stage 1)}

In Stage 1, we identified 2102 significantly differentially expressed genes between Asian and non-Asian GCs (corrected $\mathrm{p}<0.05$, online supplementary table S4). We conducted gene signature analyses using two different methodologies. First, using Ingenuity Pathway Analysis (IPA), we observed a general theme of inflammatory and immunological disease among the top enriched 'Diseases and Disorders' $(p<0.05)$ in tumours from both geographic localities (figure $3 \mathrm{~A}$, left). Within the major category 'inflammatory disease', Asian and non-Asian GCs shared several subdisease signatures (see online supplementary table S5). However, none of the constituent genes in the subdisease signatures were common between these GCs (see online supplementary table S5 and figure S5) suggesting that GCs from both localities have distinct inflammatory mechanisms. Supporting this idea, IPA analysis using 'canonical signalling pathways' revealed that only non-Asian GCs were associated with multiple signalling pathways related to T-cell biology (Fisher's exact $\mathrm{p}<0.05$; figure $3 \mathrm{~A}$, right).

Second, using Gene Set Enrichment Analysis (GSEA), we performed pathway analysis on ranked differentially expressed genes between Asian and non-Asian GCs, specifically querying genesets in the C2 (curated) and C7 (immunological) sections of the Molecular Signatures Database (MSigDB). Notably, because MSigDB geneset names are based on their original data sources, their names may sometimes obscure their actual gene content (eg, 'Rickman_Head_and_neck_Cancer_D' and 'Shedden Lung_Cancer_Good_Survival_A12' are immune-related genesets containing genes largely associated with immune function). ${ }^{23} 24$ Therefore, all enriched MSigDB genesets were manually curated to accurately reflect their specific biological processes (see online supplementary table S3).

Interrogation of C2 genesets revealed a majority (4/4) enrichment of immune-related genesets in non-Asian GCs (figure 3B; online supplementary table S6). Similarly, GSEA using C7 immunology-related genesets revealed a majority (6/8) of T-cell-related genesets enriched in non-Asian GCs, including signatures related to $\mathrm{CD} 4, \mathrm{CD} 8$ and memory T-cells (figure 3B; online supplementary table S7).

\section{Enrichment of T-cell signatures in non-Asian GCs (Stage 2)}

To confirm Stage 1, we tested whether non-Asian GCs profiled on non-Affymetrix arrays (see online supplementary table S4) were also enriched in T-cell gene signatures (Stage 2). Performing a similar analysis against the $\mathrm{C} 2$ genesets, immune signatures were again enriched in the non-Asian GCs (figure 3C, top). Likewise, enrichment of C7 T-cell-related genesets identified in Stage 1 (see online supplementary table S8) was also observed in Stage 2 non-Asian GCs (figure 3C, bottom). These results, derived from a larger series profiled on multiple 
Table 1 Clinical characteristics of expression microarray studies

\begin{tabular}{|c|c|c|c|c|c|c|c|c|c|c|}
\hline Total $n=1016$ & AMS_CDNA $(n=34)$ & $A U \_$affy $(n=68)$ & HK_CDNA ( $n=90)$ & Korea_SY ( $n=96)$ & SamsungMC $(n=432)$ & SGset1 ( $n=152)$ & SGset2 $(n=55)$ & YGC $(n=65)$ & LEEDS $(n=24)$ & p Value* (t test) \\
\hline \multicolumn{11}{|l|}{ Age (years) } \\
\hline Range & $50.0-90.5$ & $32.0-85.0$ & $35.0-88.0$ & $30.0-90.0$ & $23.0-74.0$ & 23.4-92.4 (1 missing) & $43.0-85.0$ & $32.0-83.0$ & $53.0-83.7$ & - \\
\hline Mean \pm SD & $72.2 \pm 8.8$ & $65.0 \pm 12.4$ & $68.5 \pm 11.9$ & $63.2 \pm 10.6$ & $51.9 \pm 10.7$ & $65.6 \pm 12.9$ (1 missing) & $68.7 \pm 9.1$ & $61.0 \pm 11.5$ & $72.5 \pm 8.5$ & 0.10 \\
\hline \multicolumn{11}{|l|}{ Gender (\%) } \\
\hline Female & $10(29.4)$ & $20(29.4)$ & $39(43.3)$ & $26(27.1)$ & $152(35.2)$ & $59(38.8)$ & $20(36.4)$ & $19(29.2)$ & $11(45.8)$ & 0.98 \\
\hline Male & $24(70.6)$ & $48(70.6)$ & $51(56.7)$ & $70(72.9)$ & $280(64.8)$ & $92(60.5)$ & $35(63.6)$ & $46(70.8)$ & $13(54.2)$ & 0.97 \\
\hline Missing & - & - & - & - & - & $1(0.66)$ & - & - & - & - \\
\hline \multicolumn{11}{|l|}{ Staget $(\%)$} \\
\hline 1 & $6(17.6)$ & $11(16.2)$ & $13(14.4)$ & $8(8.33)$ & $55(12.7)$ & $27(17.8)$ & $9(16.4)$ & $12(18.5)$ & $5(20.8)$ & 0.14 \\
\hline$\|$ & $7(20.6)$ & $15(22.1)$ & $19(21.1)$ & $22(22.9)$ & $160(37.0)$ & $22(14.5)$ & $12(21.8)$ & $2(3.08)$ & $4(16.7)$ & 0.96 \\
\hline III & $12(35.3)$ & $35(51.5)$ & $43(47.8)$ & $37(38.5)$ & $144(33.3)$ & $54(35.5)$ & $18(32.7)$ & $35(53.8)$ & $12(50.0)$ & 0.44 \\
\hline IV & $9(26.5)$ & $7(10.3)$ & $15(16.7)$ & $29(30.2)$ & $72(16.7)$ & $48(31.6)$ & $15(27.3)$ & $16(24.6)$ & $3(12.5)$ & 0.25 \\
\hline Missing & - & - & - & - & $1(0.23)$ & $1(0.66)$ & $1(1.82)$ & - & - & - \\
\hline \multicolumn{11}{|c|}{ Lauren's histopathology (\%) } \\
\hline Intestinal & $16(47.1)$ & $33(48.5)$ & $68(75.6)$ & $32(33.3)$ & $139(32.2)$ & $79(52.0)$ & $38(69.1)$ & $20(30.8)$ & $17(70.8)$ & 0.57 \\
\hline Diffuse & $12(35.3)$ & $29(42.6)$ & $13(14.4)$ & $21(21.9)$ & $280(64.8)$ & $56(36.8)$ & $11(20.0)$ & $31(47.7)$ & $5(20.8)$ & 0.90 \\
\hline Mixed/unclassifiable & $6(17.6)$ & $6(8.82)$ & $9(10.0)$ & $43(44.8)$ & $13(3.01)$ & $16(10.5)$ & $5(9.09)$ & $12(18.5)$ & $2(8.33)$ & 0.54 \\
\hline Missing & - & - & - & - & - & $1(0.66)$ & $1(1.82)$ & $2(3.08)$ & - & - \\
\hline \multicolumn{11}{|c|}{ Helicobacter Pylori status (\%) } \\
\hline Positive & - & $49(72.1)$ & $48(53.3)$ & - & - & $48(31.6)$ & $19(34.5)$ & - & - & N.A. \\
\hline Negative & - & $17(25.0)$ & $42(46.7)$ & - & - & $25(16.4)$ & $6(10.9)$ & - & - & N.A. \\
\hline Missing/ unknown & - & $2(2.94)$ & - & - & - & $79(52.0)$ & $30(54.5)$ & - & - & - \\
\hline \multicolumn{11}{|l|}{ Tumour location (\%) } \\
\hline Upper third & $11(32.4)$ & $19(27.9)$ & $28(31.1)$ & - & $54(12.5)$ & $15(9.87)$ & $9(16.4)$ & $4(6.15)$ & $6(25.0)$ & 0.04 \\
\hline Middle third & $15(44.1)$ & $36(52.9)$ & - & - & $115(26.6)$ & $67(44.1)$ & $11(20.0)$ & $31(47.7)$ & $9(37.5)$ & 0.26 \\
\hline Lower third & $10(29.4)$ & $12(17.6)$ & $41(45.6)$ & - & $226(52.3)$ & $37(24.3)$ & $15(27.3)$ & $27(41.5)$ & $8(33.3)$ & 0.16 \\
\hline Others $¥$ & - & $1(1.47)$ & - & - & $37(8.56)$ & $22(14.5)$ & - & $1(1.54)$ & - & N.A. \\
\hline Missing & $8(23.5)$ & - & $21(23.3)$ & - & - & $11(7.24)$ & $20(36.4)$ & $2(3.08)$ & $1(4.17)$ & - \\
\hline \multicolumn{11}{|c|}{ Tumour differentiation (\%) } \\
\hline Well & $1(2.94)$ & $2(2.94)$ & - & $12(12.5)$ & $10(2.31)$ & $5(3.29)$ & $1(1.82)$ & $20(30.8)$ & $2(8.33)$ & 0.40 \\
\hline Moderate & $10(29.4)$ & $22(32.4)$ & - & $33(34.4)$ & $108(25.0)$ & $54(35.5)$ & $23(41.8)$ & $15(23.1)$ & $12(50.0)$ & 0.52 \\
\hline Poor/undifferentiated & $22(64.7)$ & $44(64.7)$ & - & $43(44.8)$ & $186(43.1)$ & $90(59.2)$ & $29(52.7)$ & $6(9.23)$ & $10(41.7)$ & 0.24 \\
\hline Others§ & - & - & - & $8(8.33)$ & $127(29.4)$ & - & - & $20(30.8)$ & - & N.A. \\
\hline Missing & $1(2.94)$ & & - & - & $1(0.23)$ & $3(1.97)$ & $2(3.64)$ & $4(6.15)$ & - & - \\
\hline
\end{tabular}

${ }^{*} \mathrm{p}$-value from hypothesis testing between Asian and non-Asian cohorts.

tAmerican Joint Committee on Cancer (AJCC) staging 6th edition.

thostom, anastomosis, multisite, etc.

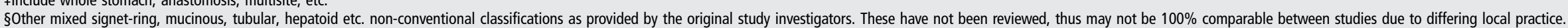




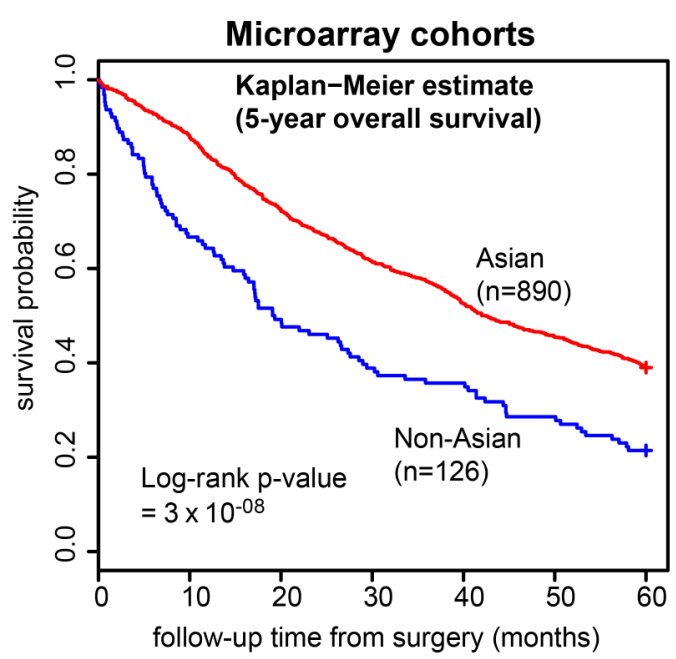

Figure 1 Five-year overall survival outcomes in the nine expression studies. Kaplan-Meier curves comparing Asian (red) versus non-Asian (blue) 5-year overall survival outcomes in the nine expression studies.

different microarray platforms, suggest a global enrichment of T-cell expression signatures in non-Asian GCs.

\section{Immunohistochemistry supports T-cell enrichment in non-Asian GCs}

To validate the immune-related gene expression differences between Asian and non-Asian GCs, we performed IHC on TMA panels comprising Asian and non-Asian GCs. Using automated image analysis to investigate quantitative and qualitative differences in intratumoural inflammatory cells and T-cells, we analysed an independent cohort of 219 Japanese GCs, and a separate cohort of 446 Caucasian GCs. Among these patients, a total of 133 were treated with chemotherapy after surgery (127 were treated with a 5 -fluorouracil (5FU)-related chemotherapy) (see online supplementary table S9).

Neutrophils and macrophages are common cells associated with inflammation. We observed that Japanese GCs had a significantly higher number of cells positive for neutrophil marker
CD66b ( $p=2 \times 10^{-05}$; figure 4A; online supplementary table $\mathrm{S} 10)$, and the pan-leukocyte marker CD45 ( $\mathrm{p}=2 \times 10^{-03}$; online supplementary figure S6 and table S10). Conversely, Caucasian GCs were significantly enriched for the macrophage marker CD68 ( $\mathrm{p}=4 \times 10^{-04}$; figure 4A; online supplementary table S10).

Supporting the gene expression microarray results, the majority of T-cell markers were significantly enriched in Caucasian compared with Japanese GCs (T-cell makers: CD3: $\mathrm{p}=4 \times 10^{-06}$; CD45R0: $p=9 \times 10^{-15} ; \mathrm{CD} 8: \mathrm{p}=6 \times 10^{-07}$; figure 4B; see online supplementary table $\mathrm{S} 10)$. The exception was the immunosuppressive T-regulatory (Treg) cell marker FOXP3 $\left(p=6 \times 10^{-08}\right.$; figure 4B; online supplementary table $\mathrm{S} 10$ ), which was significantly enriched in Japanese compared with Caucasian GCs. These results confirm the two-stage gene expression analysis that Asian and non-Asian GCs are likely to have distinct immune-related components, especially a higher abundance of T-cell infiltration in non-Asian GCs.

\section{Tumour immunity may influence location-specific survival differences}

Continuing to focus on the TMA cohorts, we then explored whether geographic location-specific survival differences might be influenced by tumour immunity factors. We performed a univariate Cox regression analysis of 5 -year overall survival with geographic locality. Subsequently, we fitted the univariate model with other factors (eg, cancer stage, age, CD3 status etc.), in turn, to assess whether these factors might influence the adjusted HR for locality effects. We found that besides 'age', only the immune cell markers (ie, CD3, CD68, CD66b, CD8, CD45R0 and FOXP3) could individually reduce the significance of geographic locality-specific prognosis (figure 5A). This suggests that, apart from 'age', differences in tumour-infiltrating immune cells may influence locality-specific survival in the cohorts. Additionally, adjustment for chemotherapy status also found locality-specific prognosis to be affected only by tumour immunity factors (see online supplementary figure S7).

A multivariate Cox regression model incorporating all significant factors (univariate Cox regression p value $<0.05$; see online supplementary figure S8A) and year of surgery, revealed that CD68 and CD3 were the only immune markers with
Figure 2 Schematic diagram of study. The study examined a total of nine microarray datasets (total $\mathrm{n}=1016$ ), comprising cohorts from Asian and non-Asian localities. For the initial assessment (Stage 1), the four Affymetrix-based studies (total $n=299$ ) were considered. Stage 2 was a validation study assessing five non-Affymetrix platform studies (total $n=717)$. Further validation was performed on two tissue microarray datasets (total $n=665$ ), comprising Caucasian and Japanese patients with gastric cancer.

\section{Study schematic diagram}

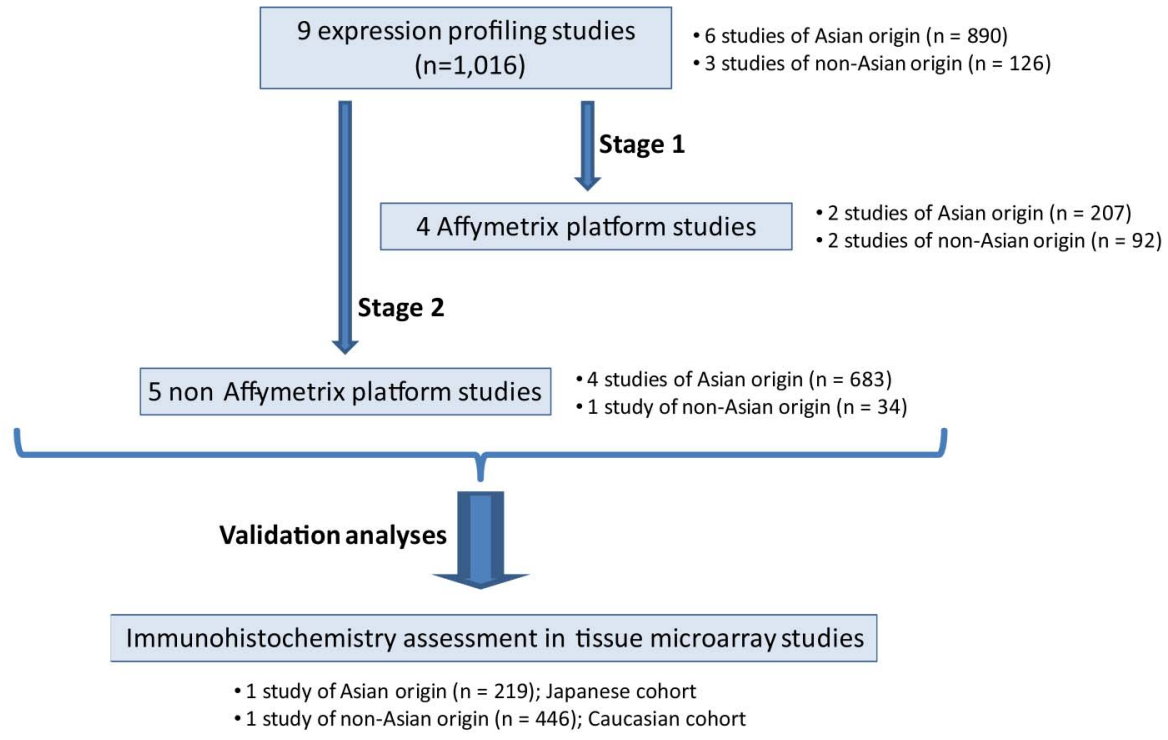

- 1 study of Asian origin ( $n=219)$; Japanese cohort 
A

\begin{tabular}{lc} 
Top 5 Diseases and Disorders & \\
\hline Name & $\mathrm{p}$-value \\
\hline Asian & $5 \times 10^{-15}-6 \times 10^{-03}$ \\
Infectious Disease & $6 \times 10^{-14}-5 \times 10^{-03}$ \\
Cancer & $6 \times 10^{-11}-5 \times 10^{-03}$ \\
Cardiovascular Disease & $6 \times 10^{-08}-5 \times 10^{-03}$ \\
Connective Tissue Disorders & $6 \times 10^{-08}-3 \times 10^{-03}$ \\
Inflammatory Disease & \\
Non-Asian & $2 \times 10^{-05}-2 \times 10^{-02}$ \\
Connective Tissue Disorders & $2 \times 10^{-05}-2 \times 10^{-02}$ \\
Skeletal and Muscular Disorders & $3 \times 10^{05}-2 \times 10^{02}$ \\
Immunological Disease & $3 \times 10^{05}-2 \times 10^{02}$ \\
Inflammatory Disease & $4 \times 10^{-05}-2 \times 10^{-02}$ \\
Neurological Disease &
\end{tabular}

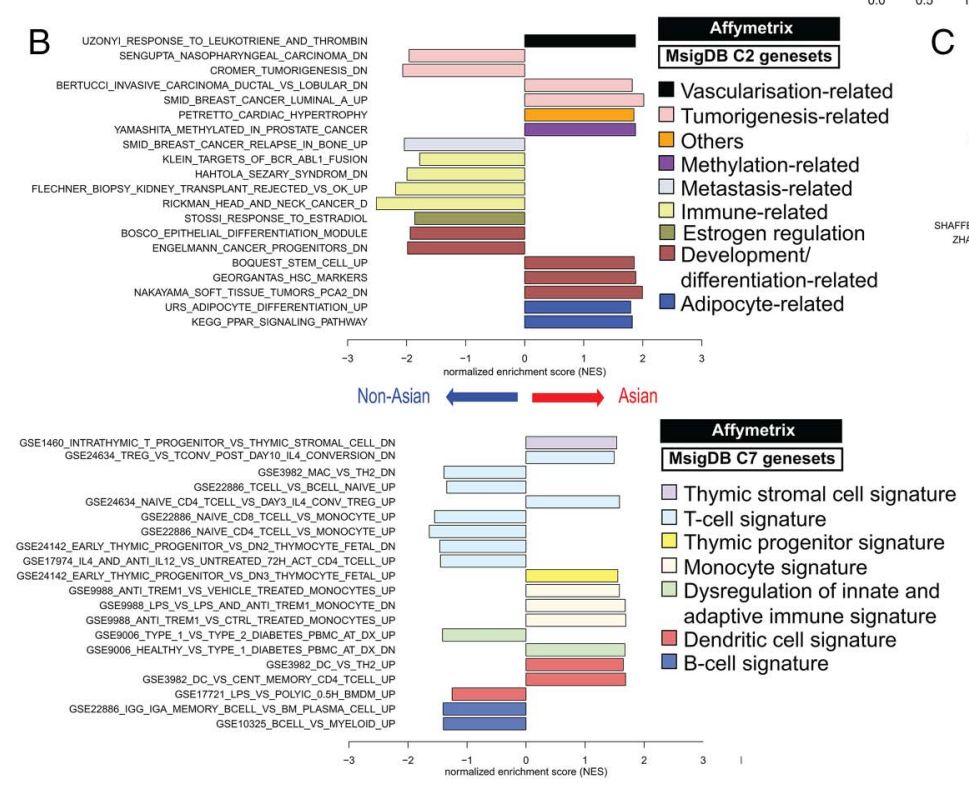

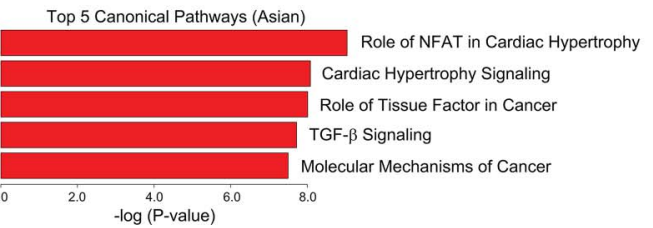

Top 5 Canonical Pathways (Non-Asian)
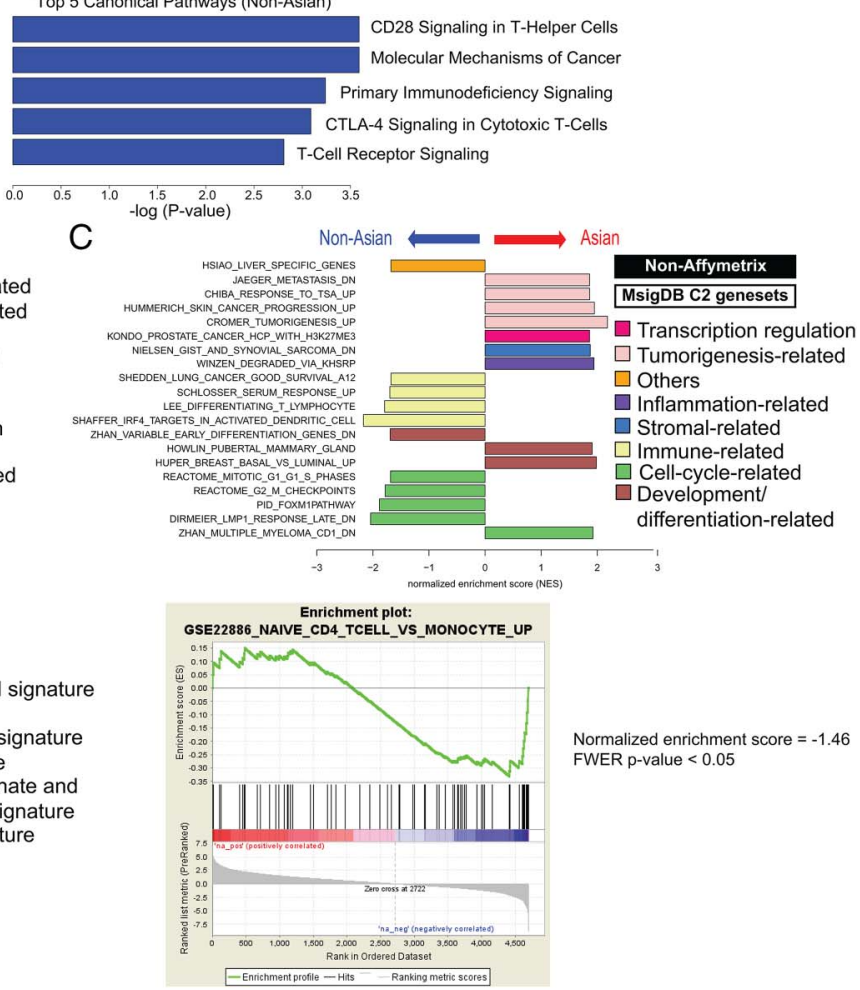

Figure 3 Pathway analyses of Asian versus non-Asian gastric cancer (GC) profiles. Panel (A) illustrates Ingenuity Pathway Analysis (IPA) results; 'inflammatory disease' is among the top five diseases and disorders most commonly associated with both Asian and non-Asian GCs. However, non-Asian GCs are also enriched for 'immunological disease'. For signalling pathways, T-cell-related canonical signalling pathways (eg, 'CD28 Signalling in T-Helper Cells', 'CTLA-4 Signalling in Cytotoxic T-Cells', 'T-Cell Receptor Signalling') feature prominently in the top five significant canonical pathways (Fisher's test $p<0.05$ ) in non-Asian tumours. Panel $(B)$ shows results from GeneSet Enrichment Analysis (GSEA) for Affymetrix-based arrays. Interrogating MSigDB C2 (curated) genesets revealed multiple immune signatures (pale yellow bars; top diagram) among the top ten enriched genesets associated with non-Asian GCs, while such signatures are absent among Asian samples. Additionally, interrogating MSigDB C7 (immunological) genesets showed that the immune signatures observed in non-Asian GCs are enriched for T-cell-related signatures (light blue bars; bottom diagram) compared with Asian GCs. Panel (C) top diagram depicts GSEA results for non-Affymetrix-based arrays, when interrogated against C2 genesets. In general, immune signatures (pale yellow bars) are also enriched among the top ten genesets associated with non-Affymetrix non-Asian GCs. The top portion of the bottom diagram shows the running enrichment score (ES) for the T-cell-related pathway 'GSE22886_NAIVE_CD4_TCELL_VS_MONOCYTE_UP'. The ES for the pathway is defined as the peak score furthest from zero. In this case, ES is significantly negative (normalised $\overline{E S}=-1.46$; Familywise error rate (FWER) $p$ value $<0.05$ ) that is, enriched in non-Asian GCs in the non-Affymetrix-based studies. This is shown in the middle portion of the plot (black vertical lines; ie, members of the geneset in order of appearance in the ranked list of genes) where most of the gene members appear after the peak score.

independent associations with survival, however, their relationship with prognosis is complex and non-linear (see online supplementary figure S8B). To explain this relationship, we extrapolated previous findings from other cancer types where tumours with very high intratumour CD68/CD3 ratios are usually highly invasive, with patients bearing such tumours typically exhibiting poor prognosis. ${ }^{25}{ }^{26}$ Indeed, in our TMA cohorts, a regression analysis of CD68/CD3 ratios with 5-year overall survival revealed that increased CD68/CD3 ratios were significantly and independently associated with worse survival outcomes $(\beta=$ $-0.141 ; \mathrm{p}$ value $=9 \times 10^{-03}$; online supplementary table S11).

We then focused on the 'high-risk' group of patients with high CD68 and low CD3 levels $(n=55)$ within the TMA cohorts and found that non-Asians tended to have significantly higher CD68/CD3 ratios compared with Asians (Welch's t-test p for trend $=0.04$; figure $5 \mathrm{~B}$ ). This observation may explain at least, in part, why non-Asians with GC generally have worse survival rates compared with Asians with GC.

To provide additional support for the influence of CD68/ CD3 ratios in GC clinical outcome, we revisited the gene expression cohorts. Due to residual confounding in the RUV-4 combined data, it was not possible to directly compare the pooled Asian to the non-Asian GCs at the single-gene level. We could, however, perform similar analyses within each dataset separately. In agreement with our results from the TMA cohorts, assessment of CD68/CD3 ratios in six of the microarray expression cohorts revealed that five cohorts also exhibited a significant trend for worse survival times with higher CD68/ CD3 ratios ( $\mathrm{p}$ for trend $<0.05$; online supplementary table $\mathrm{S} 12$ and figure S9). 
A
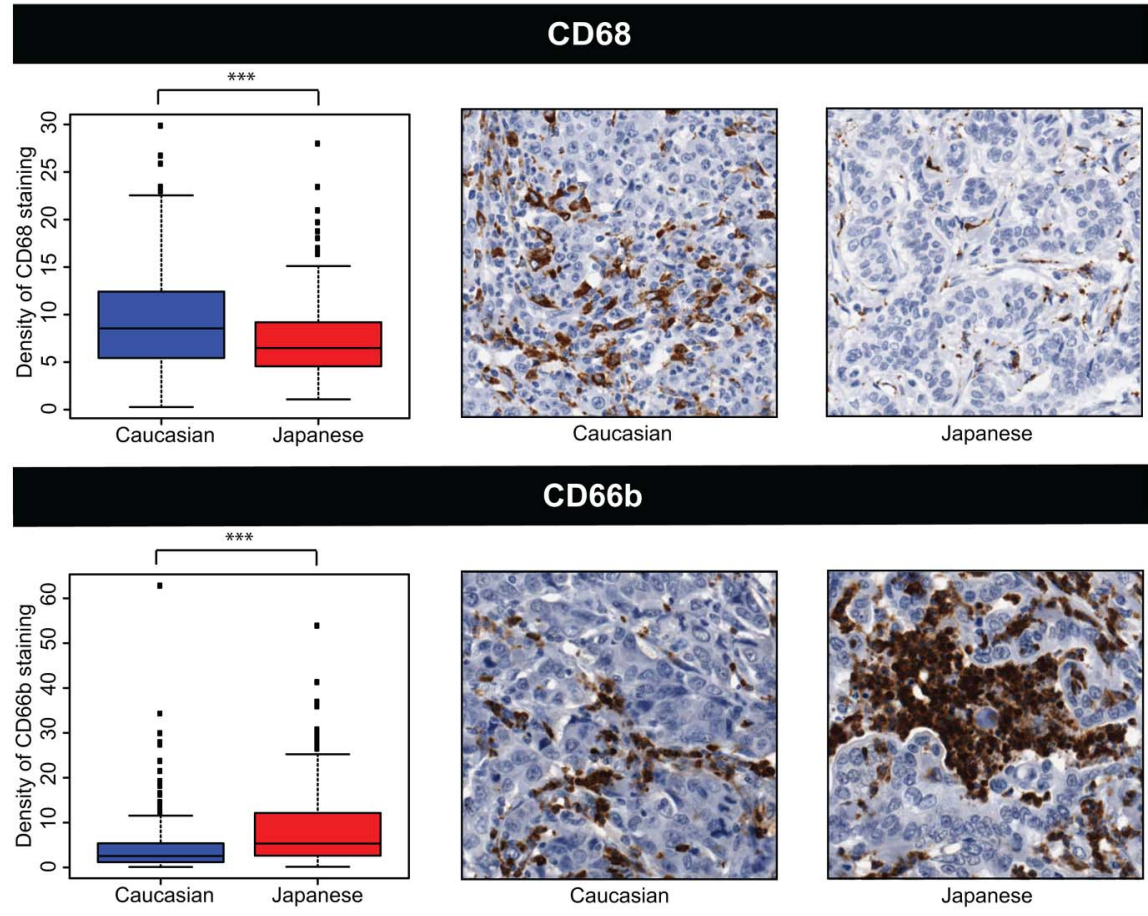

B

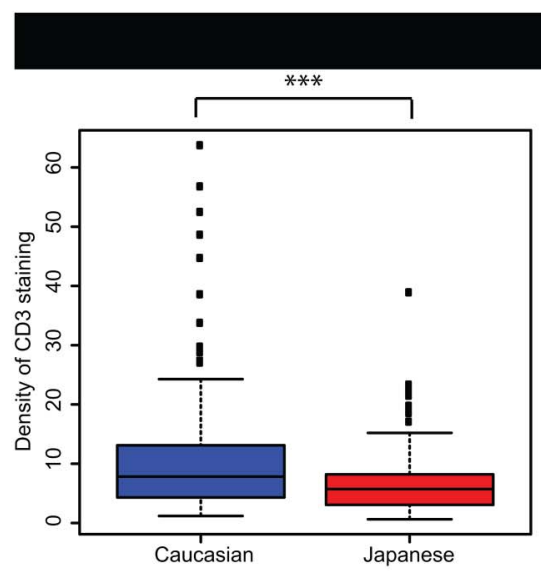

Caucasian

Japanese

\section{CD3}
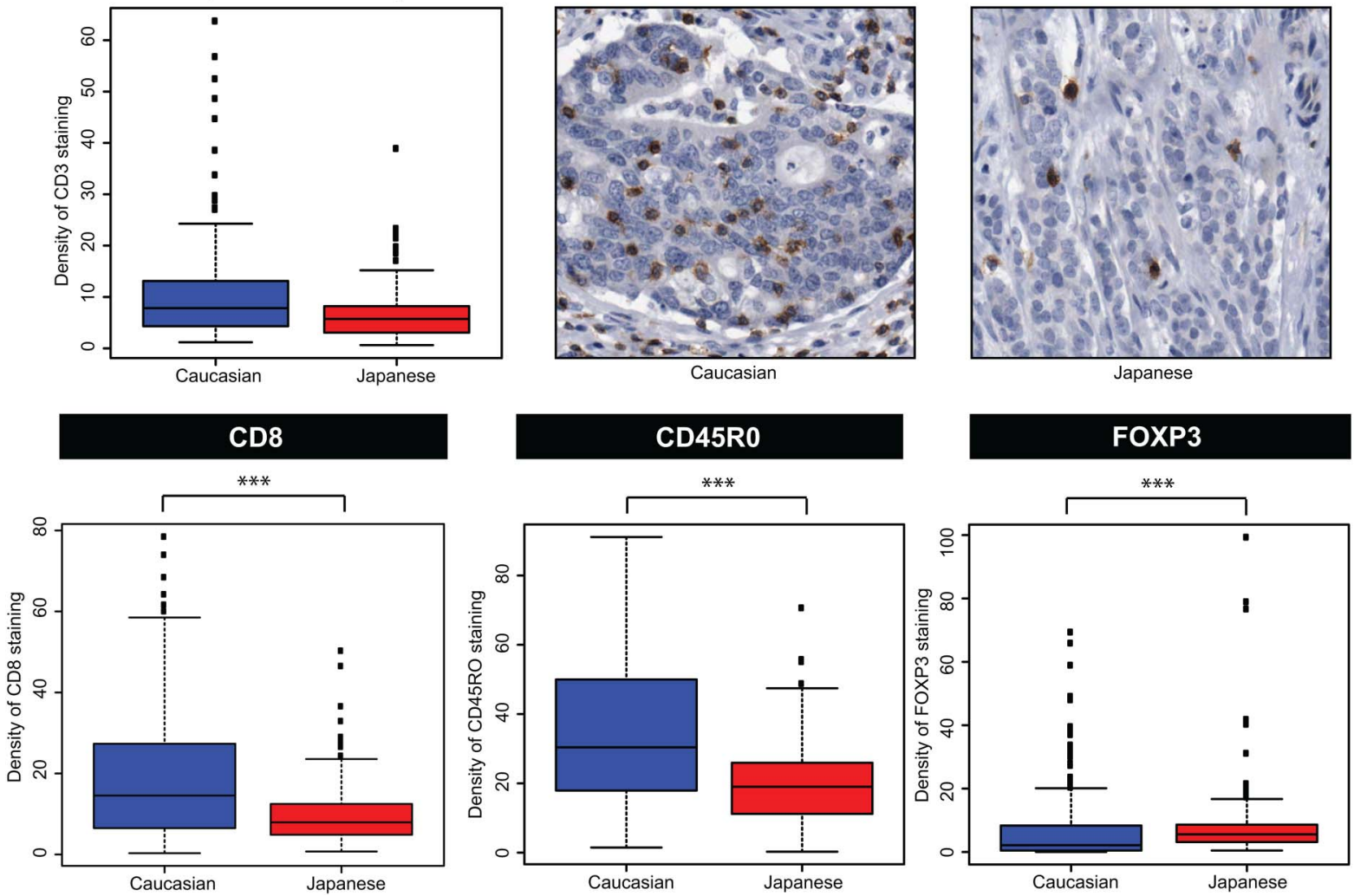

Figure 4 Immunohistochemical (IHC) assessments of immune and inflammatory cell populations in Caucasian and Japanese gastric cancer (GC) cohorts. Panel (A) depicts IHC assessment of tumour infiltration by macrophages (CD68) and neutrophils (CD66b) in Caucasian (blue) versus Japanese (red) cohorts. There is significantly more CD68 staining (top diagram) in Caucasian compared with Japanese GCs, and significantly more CD66b staining (bottom diagram) in Japanese compared with Caucasian GCs. These results suggest the presence of inflammation in both cohorts, but different cellular recruitment. Panel (B) illustrates results after quantitative IHC for T-cell-related immune markers, that is, CD3 (general T-cell marker; top diagram), CD8 (cytotoxic T-cell marker; bottom left diagram), CD45R0 (marker for memory T-cells; bottom middle diagram) and F0XP3 (marker for regulatory T-cells; bottom right diagram), in Caucasian (blue) and Japanese (red) cohorts. With the exception of FOXP3, all markers are significantly enriched in Caucasian GCs. For FOXP3, there is significantly more staining in the Japanese GC. For all cases, *** indicates a significant (Wilcoxon Mann-Whitney test $\mathrm{p}<0.05$ ) difference in the extent of staining. Panels also show corresponding IHC images from Caucasian and Japanese cohorts. 
A

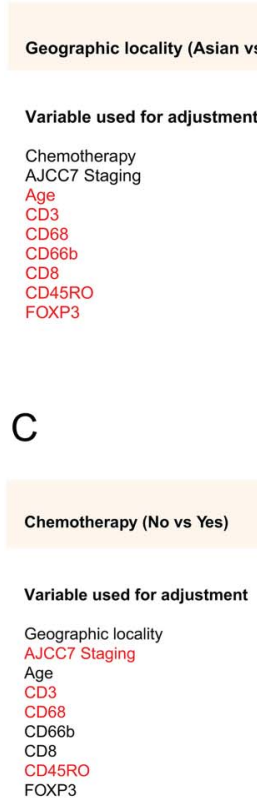

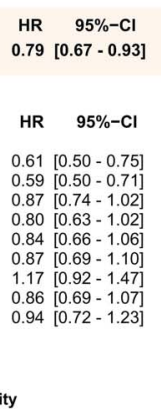

$\stackrel{0.5}{2} \stackrel{1}{2} \stackrel{2}{\text { Adjusted Hazard Ratio for geographic locality }}$

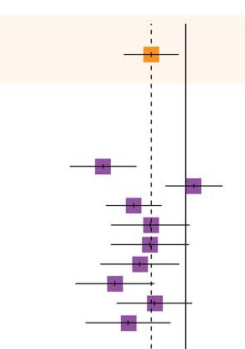

0.5

Adjusted
B

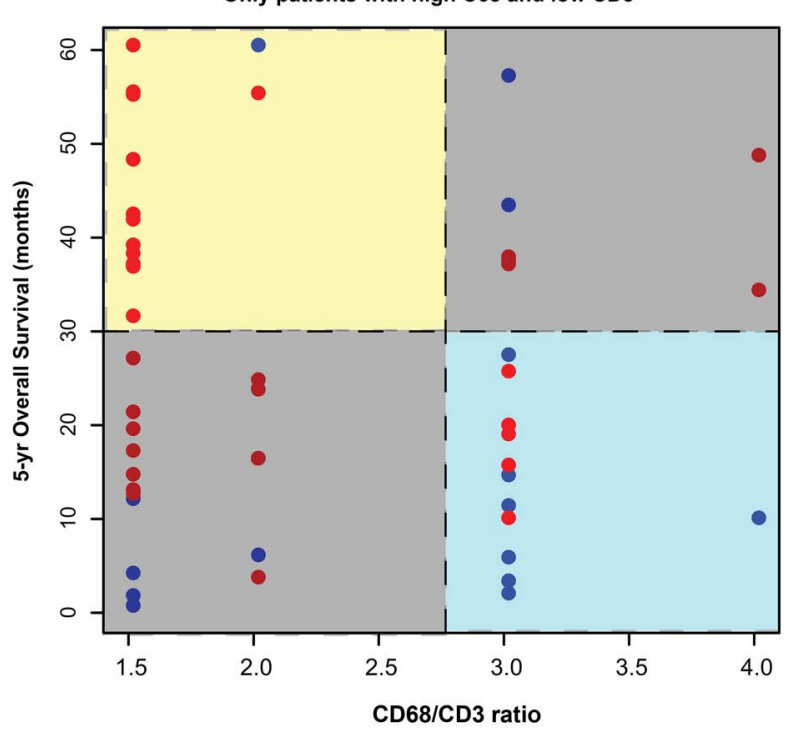

Figure 5 Assessment of effect of immune factors on geographic locality-based and chemotherapy-based survival. Panel (A) illustrates a univariate Cox model fitted with only the geographic locality factor (orange box) and fitted, in turn, with each of the respective factors (eg, chemotherapy, TNM staging, age etc). Adjusted HRs and their corresponding $\mathrm{p}$ values for locality are presented. All factors causing a change in significance of locality-specific prognosis are coloured in red text. Panel (B); scatterplot of 5-year-overall survival (months) against CD68/CD3 ratios in the tissue microarray patient group $(n=55)$ with high CD68 and low CD3 levels. There are more non-Asians (blue) than Asians (red) with higher CD68/CD3 ratios and low 5-year overall survival (light blue box). While those with lower CD68/CD3 ratios and high 5-year overall survival (light yellow box) tend to be Asians. Panel (C) illustrates a similar diagram to Panel (A), except a univariate Cox model is first fitted with the chemotherapy factor (orange box). Adjusted HRs and their corresponding $\mathrm{p}$-values for chemotherapy are presented. All factors causing a change in significance of chemotherapy outcomes are coloured in red text.

\section{GC patient outcome after chemotherapy may be influenced by tumour immunity}

Accumulating evidence suggests that antitumour activity of chemotherapy may involve the immune system. ${ }^{27}$ In an exploratory analysis, we tested whether the Asian versus non-Asian immune differences might impact on outcomes of patients treated with surgery with/without chemotherapy. In our retrospective series, patients receiving only surgery had significantly better survival than those given surgery with chemotherapythis is likely due to chemotherapy-treated patients in our series tending to have a higher Tumour Node Metastasis (TNM) stage at the time of surgery. Given this caveat, a univariate Cox regression model with/without chemotherapy was fitted. The model was then individually adjusted for various factors (eg, cancer stage, age, immune cell marker etc.). We observed that, besides cancer staging, adjusting for CD3, CD68 and CD45R0 tended to negate the survival difference between patients with or without chemotherapy. Conversely, adjusting for CD8 and FOXP3 made the survival difference more pronounced (figure $5 \mathrm{C})$. These results suggest that in GC, chemotherapy outcomes and immune effects may be interdependent.

\section{Tumour immunity differences are unlikely due to EBV infection or MMR status}

We also investigated whether the observed intratumoural immune cell differences between the Japanese and the Caucasian GC cohorts might be due to differences in EBV status or MMR status, as both factors are known to be related to high numbers of intratumoural lymphocytes. ${ }^{28-30}$ T-cells (CD8 and CD4) may also play an important role in EBV control. ${ }^{31}$ We performed RNA in situ hybridisation for EBV and compared MMR status between the Japanese $(n=219)$ and Caucasian $(n=446)$ cohorts. EBV status and MMR status levels were not significantly different between the cohorts (EBV positivity: Japanese: 6\%, Caucasian: $3 \% \chi^{2}$ test $p>0.05$; MMR deficiency: Japanese $10 \%$, Caucasian $7 \%, \chi^{2}$ test $p>0.05$; online supplementary figures S10, S11 and table S9).

\section{Association of immune markers with other clinicopathologic factors}

Finally, we assessed whether the immune markers (including CD68/CD3 ratios) might be associated with any other clinicopathologic factors (eg, pathological Tumour Node Metastasis (pTNM) stage, EBV status, MMR status etc). We found that pTNM stage was positively correlated with CD68 and CD68/CD3 ratios, while $\mathrm{EBV}$ positivity was correlated with higher $\mathrm{CD} 3, \mathrm{CD} 8$ and CD45R0 levels but negatively correlated with CD68/CD3 ratios (see online supplementary table S13). Additionally, MMR deficiency was correlated with lower CD3, CD68, FOXP3 levels, while higher tumour differentiation grade was positively correlated with all markers except CD66b, FOXP3 and CD68/CD3 ratio (see online supplementary table S13). Higher CD3, CD8 and CD68 levels were significantly correlated with diffuse-type GC (see online supplementary figure S13). Higher FOXP3 was the only marker significantly correlated with tumour location and correlated with tumours located in the middle third of the stomach 
(see online supplementary figure S13). None of the immune markers was associated with age or gender (see online supplementary table S13 and figure S14).

\section{DISCUSSION}

Our analysis of $>1600$ GCs provides evidence that Asian and non-Asian GCs may exhibit distinct gene signatures related to inflammation and immunity, with T-cell pathways preferentially associated with non-Asian GC. Our study should be regarded as hypothesis-generating requiring further validation. Due to the retrospective nature of meta-analysis studies, there is no control over the initial study design of each cohort, and confounding variables present a major issue. Our study addressed this by using a novel statistical algorithm, RUV-4, to reduce technical noise; a two-stage analysis plan for reducing platform-specific artefacts; and a gene signature-based analysis (c.f. individual genes). Moreover, although residual confounding after RUV-4 adjustment clearly remains in the gene expression data, our immunohistochemistry validation studies in independent cohorts suggest that, at least on a disease-pathway level, we have captured true biological signals.

At the gene-expression level, non-Asian GCs exhibited enrichment in multiple signatures related to T-cell biology, including CD28 and CTLA-4 signalling (figure 3). Both CD28 and CTLA-4 (cytotoxic T-lymphocyte-associated protein 4) are glycoproteins expressed by T-cells. Quantitative IHC assessment of intratumoural T-cells in independent GC samples further confirmed T-cell enrichment in Caucasian compared to Japanese GCs, such as activated (memory) CD45R0-positive T-cell populations. We also observed lower levels of FOXP3, a Treg marker, in non-Asian GCs. This is consistent with our gene expression results as Treg cells are known to have immunosuppressive functions $^{32}$ and are required for maintaining immune tolerance. ${ }^{33}$

Presently, it remains unclear why we see these immune-related differences between the cohorts. Rates of EBV infection and MMR status, both associated with GC immune cell infiltration, ${ }^{28}{ }^{29}$ were similar between the cohorts, although a larger sample size may be required for a definitive conclusion. ${ }^{34} 35$ A limitation of our study is that $H$ pylori status information was unavailable for several cohorts, precluding a correlative analysis between the immune differences and $H$ pylori exposure. $H$ pylori status is not routinely tracked in clinical management, and assessment of active exposure is also challenging since $H$ pylori exposure often occurs early in life (serology studies) ${ }^{36}$ and these organisms are usually absent in GC resection specimens. Notably, a recent study comparing US and Japanese GC patients reported that genetic variants in tumour immune checkpoint genes (eg, CTLA-4, FOXP3) may impact on clinical outcome in an ethnic-specific manner. ${ }^{37}$ It is thus possible that population differences in germline polymorphisms of immunity genes may contribute to the immune differences between GCs from different regions.

We explored whether these observed immune differences could contribute, at least in part, to differences in survival between GC patients from different regions. ${ }^{38} 39$ Our results suggest that in GC, differences in tumour immunity may indeed affect geographic locality-specific survival, similarly to what has been previously reported for other tumour types. For example, in bladder and breast cancer, high $\mathrm{CD} 68 / \mathrm{CD} 3$ or $\mathrm{CD} 3 / \mathrm{CD} 20$ ratios have been associated with worse prognosis. ${ }^{25} 26$ Assessment of CD68/CD3 ratios within our TMA and expression microarray cohorts yielded a similar conclusion. High tumour stage and EBV positivity, factors known to be associated with worse ${ }^{40}$ and better ${ }^{41}$ prognosis in GC patients, respectively, have also been found to be positively and negatively correlated with CD68/CD3, respectively. Notably, non-Asians in the highrisk CD68 high/CD3 low group tended to have significantly higher CD68/CD3 ratios compared with Asians, consistent with non-Asian GC patients having a generally worse prognosis. In the case of chemotherapy, previous studies have shown that $5 \mathrm{FU}$ can activate immune regulatory cells to stimulate protumuorigenic cytokines which can impact therapeutic efficacy. ${ }^{42}$ For patients in our cohorts given mainly 5FU-related-therapy (eg, S-1 or UFT), we found that tumour immunity can affect prognosis after chemotherapy.

The observation of immune-related differences in Asian and non-Asian GCs may potentially explain part of the geographical differences seen in recent clinical trials (eg, AVAGAST, ${ }^{7}$ RAINBOW (A Study of Paclitaxel With or Without Ramucirumab (IMC-1211B) in Metastatic Gastric Adenocarcinoma), ${ }^{43}$ LOGiC). ${ }^{8}$ Two of these trials used drugs targeting angiogenesis (ie, AVAGAST-VEGF; RAINBOW-VEGFR2), and immune cells (eg, CD68 macrophages, CD4 T-cells) have been shown to have pro-angiogenic ${ }^{44} 45$ effects, while antigen-presenting cells can inhibit angiogenic activity through cytokines (eg, IFN (interferon), IL-12 and IL-27). ${ }^{46} 47$ It will be interesting to assess whether infiltrating T-cells represent an 'immune biomarker' for the efficacy of anti-angiogenic agents. ${ }^{48} 49$

Our results may also have relevance for future trials investigating immunotherapy such as antibodies targeted at T-cell regulatory molecules (eg, CTLA-4, PD-1 and PD-L1). ${ }^{50}$ Our study identified enriched CTLA-4 signalling pathway in non-Asian GCs. CTLA-4 is the target of the anti-CTLA-4 antibody ipilimumab, and in GC, a Phase II clinical trial evaluating the role of CTLA-4 antibodies has recently been initiated (NCT01585987). ${ }^{51}$ For such therapies, geographical differences in the underlying tumour-immune biologies may affect treatment outcomes and hence the ultimate success of global trials.

In conclusion, the current study was performed on the largest collection of retrospectively sourced GC expression data, and revealed immune signature differences between Asian and non-Asian GCs. Due to the retrospective nature of this study, our results need to be further validated, perhaps through a prospective study designed to study locality effects. If confirmed, our study highlights the importance of identifying and validating novel targets and biomarkers in a geographically specific manner, to personalise interventions given to specific patient groups.

\section{Author affiliations}

${ }^{1}$ Department of Cancer Therapeutics and Stratified Oncology, Genome Institute of Singapore, Singapore, Singapore

${ }^{2}$ Department of Statistics, University of California at Berkeley, Berkeley, California, USA

${ }^{3}$ Department of Medical Oncology, National Cancer Centre, Singapore, Singapore ${ }^{4}$ Section of Pathology and Tumour Biology, Leeds Institute of Cancer and Pathology, University of Leeds, Leeds, UK

${ }^{5}$ Department of Pathology, Free University Medical Center Amsterdam, Amsterdam, The Netherlands

${ }^{6}$ Department of Internal Medicine, Yonsei Cancer Center, Seoul, South Korea

${ }^{7}$ Division of Cancer Medicine, Department of Systems Biology, MD Anderson Cancer Center, Houston, Texas, USA

${ }^{8}$ Department of Surgery, Yonsei University College of Medicine, Seoul, South Korea ${ }^{9}$ Department of Gastrointestinal Surgery, Kanagawa Cancer Center Hospital,

Yokohama, Japan

${ }^{10}$ Molecular Pathology and Genetics Division, Kanagawa Cancer Center Research Institute, Yokohama, Japan

${ }^{11}$ Gastroenterological Center, Yokohama City University Medical Center, Yokohama, Japan

${ }^{12}$ Departments of Gastrointestinal Medical Oncology, MD Anderson Cancer Center, Houston, USA

${ }^{13}$ Cancer Genomics and Biochemistry Laboratory, Peter MacCallum Cancer Centre, East Melbourne, Victoria, Australia 
${ }^{14}$ Department of Medicine Royal Melbourne Hospital, University of Melbourne, Parkville, Victoria, Australia

${ }^{15}$ Department of Medicine, National University Health System, Singapore, Singapore

${ }^{16}$ Department of Gastroenterology and Hepatology, National University Health

System, Singapore, Singapore

${ }^{17}$ National University Cancer Institute, Singapore, Singapore

${ }^{18}$ Department of Surgery, National University Health System, Singapore, Singapore

${ }^{19}$ Department of Medicine, Division of Haematology-Oncology, Samsung Medical

Centre, Sungkyunkwan University School of Medicine, Seoul, South Korea

${ }^{20}$ Department of Surgery, Gastric Cancer Center, Samsung Medical Center,

Sungkyunkwan University School of Medicine, Seoul, South Korea

${ }^{21}$ Department of Pathology, Kanagawa Cancer Center Hospital, Yokohama, Japan

${ }^{22}$ Department of Pathology, Tokyo Metropolitan Geriatric Hospital and Institute of Gerontology, Tokyo, Japan

${ }^{23}$ Department of Pathology, Maastricht University Medical Center, Maastricht, The Netherlands

${ }^{24}$ GROW-School for Oncology and Developmental Biology, Maastricht University

Medical Center, Maastricht, Netherlands

${ }^{25}$ Bioinformatics Division, Walter and Eliza Hall Institute, Victoria, Australia

${ }^{26}$ Department of Mathematics and Statistics, University of Melbourne, Australia

${ }^{27}$ Cancer and Stem Cell Biology Program, Duke-NUS Graduate Medical School,

Singapore, Singapore

${ }^{28}$ Cancer Science Institute of Singapore, National University of Singapore, Singapore, Singapore

${ }^{29}$ Cellular and Molecular Research, National Cancer Centre, Singapore, Singapore

Acknowledgements We acknowledge the technical assistance by Alex Wright (Leeds) for the automated image analysis. PT and HG contributed equally to this study. This work was supported by NMRC Singapore grants TCR/009-NUHS/2013 (PT), core grant support from Genome Institute of Singapore (PT); Association of Clinical Pathologists (SE); Pathological Society of Great Britain and Ireland (HG, TA); The Great Britain Sasakawa Foundation (HG, TY); Non-Profit Organisation Kanagawa Standard Anti-cancer Therapy Support System (AT, TY); NIH USA (R01CA150229) grant support to JL; and an NHMRC Australia Fellowship to TS.

Contributors PT and HG led the study, and were involved in its conception, design, data collection and assembly, analysis and manuscript writing. SL was involved in the study conception, design, data analysis and manuscript writing. JG and TS were involved in data analysis and manuscript writing. All other authors were involved in data collection and assembly, and manuscript writing. All authors were involved in proof-reading and gave final approval of the manuscript.

Competing interests The authors declare no competing interests.

Ethics approval Institutional Research Ethics Committees.

Provenance and peer review Not commissioned; externally peer reviewed.

Data sharing statement There is no additional unpublished data from this study. Queries regarding the study data should be directed to the Corresponding Author.

Open Access This is an Open Access article distributed in accordance with the Creative Commons Attribution Non Commercial (CC BY-NC 4.0) license, which permits others to distribute, remix, adapt, build upon this work non-commercially, and license their derivative works on different terms, provided the original work is properly cited and the use is non-commercial. See: http://creativecommons.org/ licenses/by-nc/4.0/

\section{REFERENCES}

1 Ferlay J, Soerjomataram I, Ervik $\mathrm{M}$, et al. Cancer Incidence and Mortality Worldwide: IARC CancerBase No. 112013.

2 Greenlee RT, Hill-Harmon MB, Murray T, et al. Cancer statistics, 2001. CA Cancer J Clin 2001;51:15-36.

3 Lee WJ, Lee WC, Houng SJ, et al. Survival after resection of gastric cancer and prognostic relevance of systematic lymph node dissection: twenty years experience in Taiwan. World J Surg 1995;19:707-13.

4 Mok YJ, Koo BW, Whang CW, et al. Cancer of the stomach: a review of two hospitals in Korea and Japan. World J Surg 1993;17:777-82.

5 Fuchs CS, Mayer RJ. Gastric carcinoma. N Engl J Med 1995;333:32-41.

6 Hundahl SA, Phillips JL, Menck HR. The National Cancer Data Base Report on poor survival of U.S. gastric carcinoma patients treated with gastrectomy: fifth edition American Joint Committee on Cancer staging, proximal disease, and the "different disease" hypothesis. Cancer 2000;88:921-32.

7 Ohtsu A, Shah MA, Van Cutsem E, et al. Bevacizumab in combination with chemotherapy as first-line therapy in advanced gastric cancer: a randomised, double-blind, placebo-controlled phase III study. J Clin Oncol 2011;29:3968-76.

8 Hecht JR, Bang YJ, Qin S, et al. Lapatinib in combination with capecitabine plus oxaliplatin (CapeOx) in HER2-positive advanced or metastatic gastric, esophageal, or gastroesophageal adenocarcinoma (AC): The TRIO-013/LOGiC Trial. [abstract] J Clin Oncol 2013;31(Suppl):LBA4001.
9 Azuma T. Helicobacter pylori CagA protein variation associated with gastric cancer in Asia. J Gastroenterol 2004:39:97-103.

10 Xue $\mathrm{H}$, Lin B, Ni P, et al. Interleukin-1B and interleukin-1 RN polymorphisms and gastric carcinoma risk: a meta-analysis. J Gastroenterol Hepatol 2010;25:1604-17.

11 Crew KD, Neugut Al. Epidemiology of gastric cancer. World J Gastroenterol 2006;12:354-62.

12 Deng N, Goh LK, Wang $\mathrm{H}$, et al. A comprehensive survey of genomic alterations in gastric cancer reveals systematic patterns of molecular exclusivity and co-occurrence among distinct therapeutic targets. Gut 2012;61:673-84.

13 Dulak AM, Schumacher SE, van Lieshout J, et al. Gastrointestinal adenocarcinomas of the esophagus, stomach, and colon exhibit distinct patterns of genome instability and oncogenesis. Cancer Res 2012;72:4383-93.

14 van Grieken NC, Aoyama T, Chambers PA, et al. KRAS and BRAF mutations are rare and related to DNA mismatch repair deficiency in gastric cancer from the East and the West: results from a large international multicentre study. $\mathrm{Br} J$ Cancer 2013;108:1495-501.

15 Su X, Zhan P, Gavine PR, et al. FGFR2 amplification has prognostic significance in gastric cancer: results from a large international multicentre study. $\mathrm{Br} J$ Cancer 2014;110:967-75.

16 Ossandon FJ, Villarroel C, Aguayo F, et al. In silico analysis of gastric carcinoma Serial Analysis of Gene Expression libraries reveals different profiles associated with ethnicity. Mol Cancer 2008; 7:22.

17 Gagnon-Bartsch JA, Laurent J, and Speed TP. Removing Unwanted Variation from High Dimensional Data with Negative Controls. 2013. http://statistics.berkeley.edu/ tech-reports/820

18 Gagnon-Bartsch JA, Speed TP. Using control genes to correct for unwanted variation in microarray data. Biostatistics 2012;13:539-52.

19 Smyth GK. Linear models and empirical bayes methods for assessing differentia expression in microarray experiments. Stat App/ Genet Mol Biol 2004;3:Article3.

20 Wright A, Hutchins G, Randell R, et al. TMAi (Tissue MicroArray informatics): Using virtual slides and open standards to provide usability and interoperability for tissue microarrays in clinical trials. Liverpool, UK: 6th NCRI Cancer Conference, National Cancer Research Institute, 2010.

21 Zur Hausen A, van Rees BP, van Beek J, et al. Epstein-Barr virus in gastric carcinomas and gastric stump carcinomas: a late event in gastric carcinogenesis. J Clin Pathol 2004;57:487-91.

22 Bouliotis G, Billingham L. Crossing survival curves: alternatives to the log-rank test. Trials 2011:12(Suppl 1):A137.

23 Rickman DS, Millon R, De Reynies A, et al. Prediction of future metastasis and molecular characterization of head and neck squamous-cell carcinoma based on transcriptome and genome analysis by microarrays. Oncogene 2008;27:6607-22.

24 Shedden K, Taylor JM, Enkemann SA, et al. Gene expression-based survival prediction in lung adenocarcinoma: a multi-site, blinded validation study. Nat Med 2008;14:822-7.

25 Sjodahl G, Lovgren $\mathrm{K}$, Lauss $\mathrm{M}$, et al. Infiltration of CD3 and CD68 cells in bladder cancer is subtype specific and affects the outcome of patients with muscle-invasive tumours. Urol Oncol 2014;32:791-7.

26 Eiro N, Pidal I, Fernandez-Garcia B, et al. Impact of CD68/(CD3+CD20) ratio at the invasive front of primary tumours on distant metastasis development in breast cancer. PLOS ONE 2012;7:e52796.

27 Bracci L, Schiavoni G, Sistigu A, et al. Immune-based mechanisms of cytotoxic chemotherapy: implications for the design of novel and rationale-based combined treatments against cancer. Cell Death Differ 2014;21:15-25.

28 Grogg KL, Lohse CM, Pankratz VS, et al. Lymphocyte-rich gastric cancer: associations with Epstein-Barr virus, microsatellite instability, histology, and survival. Mod Pathol 2003;16:641-51.

29 Leung SY, Yuen ST, Chung LP, et al. Microsatellite instability, Epstein-Barr virus, mutation of type II transforming growth factor beta receptor and BAX in gastric carcinomas in Hong Kong Chinese. Br J Cancer 1999;79:582-8.

30 Nakamura S, Ueki T, Yao T, et al. Epstein-Barr virus in gastric carcinoma with lymphoid stroma. Special reference to its detection by the polymerase chain reaction and in situ hybridization in 99 tumours, including a morphologic analysis. Cancer 1994;73:2239-49.

31 Landais $E$, Saulquin $X$, Houssaint $E$. The human $T$ cell immune response to Epstein-Barr virus. Int J Dev Biol 2005;49:285-92.

32 Jie HB, Gildener-Leapman N, Li J, et al. Intratumoural regulatory T cells upregulate immunosuppressive molecules in head and neck cancer patients. Br J Cancer 2013; 109:2629-35.

33 Sakaguchi S, Yamaguchi T, Nomura T, et al. Regulatory T cells and immune tolerance. Cell 2008;133:775-87.

34 Takada K. Epstein-Barr virus and gastric carcinoma. Mol Pathol 2000;53:255-61.

35 Camargo MC, Murphy G, Koriyama C, et al. Determinants of Epstein-Barr virus-positive gastric cancer: an international pooled analysis. $\mathrm{Br} J$ Cancer 2011;105:38-43

36 Perry S, de la Luz Sanchez M, Yang $S$, et al. Gastroenteritis and transmission of Helicobacter pylori infection in households. Emerg Infect Dis 2006;12:1701-8. 
37 Sunakawa Y, Volz NB, Zhang W, et al. Genetic variants in tumour immune checkpoints as prognostic markers in patients (pts) with localised advanced gastric cancer (AGC). [abstract] J Clin Oncol 2014;32:5s(Suppl):4018.

38 Roukos DH, Kappas AM. Perspectives in the treatment of gastric cancer. Nat Clin Pract Oncol 2005;2:98-107.

39 Bickenbach K, Strong VE. Comparisons of gastric cancer treatments: East vs. West. J Gastric Cancer 2012;12:55-62.

40 Howlader N, Noone AM, Krapcho M, et al. SEER cancer statistics review, 19752011. Bethesda, MD: National Cancer Institute. http://seer.cancer.gov/csr/1975_ 2011/, based on November 2013 SEER data submission, posted to the SEER web site, April 2014.

41 Camargo MC, Kim WH, Chiaravalli AM, et al. Improved survival of gastric cancer with tumour Epstein-Barr virus positivity: an international pooled analysis. Gut 2014;63:236-43.

42 Bruchard M, Mignot G, Derangere V, et al. Chemotherapy-triggered cathepsin B release in myeloid-derived suppressor cells activates the Nlrp3 inflammasome and promotes tumour growth. Nat Med 2013;19:57-64.

43 Wilke H, Van Cutsem E, Oh SC, et al. RAINBOW: A global, phase III, randomised, double-blind study of ramucirumab plus paclitaxel versus placebo plus paclitaxel in the treatment of metastatic gastroesophageal junction (GEJ) and gastric adenocarcinoma following disease progression on first-line platinum- and fluoropyrimidine-containing combination therapy rainbow IMCL CP12-0922 (I4T-IE-JVBE). [abstract] J Clin Oncol 2014;32(Suppl 3):LBA7.
44 Mantovani A, Schioppa T, Porta C, et al. Role of tumour-associated macrophages in tumour progression and invasion. Cancer Metastasis Rev 2006;25:315-22.

45 Zou W, Restifo NP. T(H)17 cells in tumour immunity and immunotherapy. Nat Rev Immunol 2010;10:248-56.

46 Dinney $C P$, Bielenberg DR, Perrotte $P$, et al. Inhibition of basic fibroblast growth factor expression, angiogenesis, and growth of human bladder carcinoma in mice by systemic interferon-alpha administration. Cancer Res 1998;58:808-14.

47 Shimizu M, Shimamura M, Owaki T, et al. Antiangiogenic and antitumour activities of IL-27. J Immunol 2006;176:7317-24.

48 Nikolinakos PG, Altorki N, Yankelevitz D, et al. Plasma cytokine and angiogenic factor profiling identifies markers associated with tumour shrinkage in early-stage non-small cell lung cancer patients treated with pazopanib. Cancer Res 2010;70:2171-9.

49 Shojaei F, Wu X, Malik AK, et al. Tumour refractoriness to anti-VEGF treatment is mediated by CD11b+Gr1+ myeloid cells. Nature Biotechnol 2007;25:911-20.

50 Topalian SL, Hodi FS, Brahmer JR, et al. Safety, activity, and immune correlates of anti-PD-1 antibody in cancer. N Engl J Med 2012;366:2443-54.

51 Moehler MH, Kim YH, Tan IB, et al. Sequential ipilimumab (Ipi) versus best supportive care (BSC) following first-line chemotherapy (Ctx) in patients (pts) with unresectable locally advanced or metastatic gastric or gastro-esophageal junction (GEJ) cancer: A randomised, open-label, two-arm, phase II trial (CA184-162) of immunotherapy as a maintenance concept. [abstract] J Clin Oncol 2013;31(Suppl): TPS4151. 